Internat. J. Math. \& Math. Sci.

Vol. 23, No. 5 (2000) 297-311

S0161171200000880

(C) Hindawi Publishing Corp.

\title{
ON THE EXISTENCE OF A SOLUTION FOR SOME DISTRIBUTED OPTIMAL CONTROL HYPERBOLIC SYSTEM
}

\author{
DARIUSZ IDCZAK and STANISŁAW WALCZAK
}

(Received 12 December 1994 and in revised form 30 March 1995)

\begin{abstract}
We consider a Bolza problem governed by a linear time-varying DarbouxGoursat system and a nonlinear cost functional, without the assumption of the convexity of an integrand with respect to the state variable. We prove a theorem on the existence of an optimal process in the classes of absolutely continuous trajectories of two variables and measurable controls with values in a fixed compact and convex set.
\end{abstract}

Keywords and phrases. Distributed parameters system, optimal control, existence theorem, Darboux-Goursat system.

2000 Mathematics Subject Classification. Primary 49J20; Secondary 26B30, 49 J45.

1. Introduction. Let us consider a control system described by a system of ordinary differential equations of the form

$$
\dot{x}=f(t, x, u), \quad x(0)=x_{0}, \quad x(1)=x_{1},
$$

with a cost functional

$$
I(x, u)=\int_{0}^{1} f^{0}(t, x, u) d t, \quad u \in M
$$

where $f:[0,1] \times \mathbb{R}^{n} \times M \rightarrow \mathbb{R}^{n}, f^{0}:[0,1] \times \mathbb{R}^{n} \times M \rightarrow \mathbb{R}, M$ is some subset of the space $\mathbb{R}^{r}$.

One of the fundamental problems of optimization theory is the question of the existence of optimal processes for the system of (1.1) and (1.2). This problem was the topic of investigations in many papers and monographs (cf. [1, 5] and the references therein). The natural spaces in which one studies the existence of solutions for the system (1.1) and (1.2) are the space of absolutely continuous trajectories $A C\left([0,1], \mathbb{R}^{n}\right)$ and the space of essentially bounded controls with values in the set $M$. Under some assumptions about the functions $f, f^{0}$, and the set $M$ (the growth conditions of the function $f^{0}$, the convexity of $f^{0}$ with respect to $u$ as well as the convexity and the compactness of $M$ ), it is possible to prove that the system (1.1) and (1.2) possesses a solution in the space $A C\left([0,1], \mathbb{R}^{n}\right) \times L^{\infty}\left([0,1], \mathbb{R}^{r}\right)(\mathrm{cf} .[1,5])$.

In the present paper, we consider the problem of the existence of solutions for a system with distributed parameters of the form 


$$
\begin{gathered}
\frac{\partial^{2} z}{\partial x \partial y}=A_{0}(x, y) z+A_{1}(x, y) \frac{\partial z}{\partial x}+A_{2}(x, y) \frac{\partial z}{\partial y}+B(x, y) u \quad \text { a.e. on } K, \\
z(\cdot, 0) \equiv 0 \quad \text { on }[0,1], \quad z(0, \cdot) \equiv 0 \quad \text { on }[0,1]
\end{gathered}
$$

with a cost functional

$$
I(z, u)=\iint_{K} f^{0}(x, y, z(x, y), u(x, y)) d x d y, \quad u \in M,
$$

where $z=\left(z^{1}, \ldots, z^{n}\right), u=\left(u^{1}, \ldots, u^{r}\right),(x, y) \in K=[0,1] \times[0,1], f^{0}: K \times \mathbb{R}^{n} \times$ $\mathbb{R}^{r} \rightarrow \mathbb{R}, M \subset \mathbb{R}^{r}$ is a convex and compact set. Control system (1.3) and (1.4) is considered in the space of trajectories which are absolutely continuous on $K(z \in A C)$ (cf. [11]) and in the space $u_{M}$ of controls $u$ essentially bounded and such that $u(x, y) \in$ $M$ for $(x, y) \in K$ a.e.

The basic result of our paper is a theorem on the existence of solutions, stating that if the function $f^{0}$ is convex with respect to $u$, continuous with respect to $(z, u)$, measurable with respect to $(x, y)$, and satisfies some growth condition, then the system (1.3), (1.4), and (1.5) possesses an optimal solution. This theorem has a form quite analogous to existence theorems for ordinary systems.

Systems of the form (1.3) were the objects of investigations in many papers. Essential results concerning the existence of smooth solutions can be found in [2]. The problem of the existence of solutions in Sobolev spaces is considered in [9]. In [3, 8], the existence and uniqueness of a solution in the class of continuous functions is assumed. Under the above assumptions, the maximum principle for piecewise continuous controls is proved. In [13], the system (1.3) and (1.4), with a cost functional of the form

$$
\begin{aligned}
I(z, u)= & \int_{0}^{1} \int_{0}^{1}\left(c_{0}(x, y) z(x, y)+c_{1}(x, y) \frac{\partial z}{\partial x}(x, y)\right. \\
& \left.+c_{2}(x, y) \frac{\partial z}{\partial y}(x, y)+d(x, y) u(x, y)\right) d x d y \\
+ & \int_{0}^{1}\left(e_{1}(x) z(x, 1)+e_{2}(x) \frac{\partial z}{\partial x}(x, 1)\right) d x \\
+ & \int_{0}^{1}\left(e_{3}(y) z(1, y)+e_{4}(x) \frac{\partial z}{\partial y}(1, y)\right) d y
\end{aligned}
$$

is considered in the spaces of absolutely continuous trajectories and measurable controls with values in a fixed compact and convex subset of $\mathbb{R}^{r}$. Using DubovitskiiMilyutin method, the author gives necessary conditions for optimality that are analogous to the Pontryagin maximum principle for ordinary systems.

In our paper, we introduce the notion of equiabsolute continuity of a family of absolutely continuous functions of two variables and give necessary and sufficient conditions for such a family to be equiabsolutely continuous (the analogue of [1, 10.2(i)]). Next, we prove the Ascoli-Arzela theorem for absolutely continuous functions of two variables. Making use of this theorem, we prove an analogue of $[1,10.8(\mathrm{iv})]$ for system (1.3). Finally, on the basis of the lower semicontinuity theorem (cf. [1, 10.8(i)]), we obtain a theorem on the existence of an optimal solution of problem (1.3), (1.4), and (1.5). 
Systems of the form (1.3), (1.4), and (1.5) have a natural physical interpretation which is given at the end of this paper.

2. Preliminaries. First, we recall the definition of an absolutely continuous function on $K$, introduced in [11].

DEFinITION 2.1. A function $z: K \rightarrow \mathbb{R}$ is called an absolutely continuous function on $K$ (shortly, an $A C$ function) if the associated function $F_{z}$ of an interval is an absolutely continuous function of an interval and the functions $z(\cdot, 0), z(0, \cdot)$ are absolutely continuous functions of one variable on $[0,1]$.

The associated function $F_{z}$ of an interval is defined by the formula

$$
F_{z}\left(\left[x_{1}, x_{2}\right] \times\left[y_{1}, y_{2}\right]\right)=z\left(x_{2}, y_{2}\right)-z\left(x_{1}, y_{2}\right)-z\left(x_{2}, y_{1}\right)+z\left(x_{1}, y_{1}\right)
$$

for all intervals $\left[x_{1}, x_{2}\right] \times\left[y_{1}, y_{2}\right] \subset K$.

Let us recall that a function $F$ of an interval $Q \subset K$ is called absolutely continuous if, for any $\varepsilon>0$, there exists $\delta>0$ such that $\sum_{i=1}^{N}\left|F\left(Q_{i}\right)\right|<\varepsilon$ for all finite systems of nonoverlapping closed intervals $Q_{i} \subset K, i=1,2, \ldots, N$, such that $\sum_{i=1}^{N} \mu_{2}\left(Q_{i}\right)<\delta$, where $\mu_{2}$ denotes Lebesgue measure in $K$ (cf. [6]).

In [11], it was shown that $z: K \rightarrow \mathbb{R}$ is absolutely continuous if and only if there exist integrable functions $l^{1,2} \in L^{1}(K, \mathbb{R}), l^{1}, l^{2} \in L^{1}([0,1], \mathbb{R})$, and a constant $c \in \mathbb{R}$ such that

$$
z(x, y)=\int_{0}^{x} \int_{0}^{y} l^{1,2}+\int_{0}^{x} l^{1}+\int_{0}^{y} l^{2}+c
$$

for all $(x, y) \in K$.

Making use of the above integral representation, we can demonstrate that the absolutely continuous function $z$ possesses (in the classical sense) the partial derivatives

$$
\frac{\partial z}{\partial x}=\int_{0}^{y} l^{1,2}+l^{1}, \quad \frac{\partial z}{\partial y}=\int_{0}^{x} l^{1,2}+l^{2}, \quad \frac{\partial^{2} z}{\partial x \partial y}=l^{1,2}
$$

defined for $(x, y) \in K$ a.e. These derivatives are, of course, integrable on $K$.

A vector function $z=\left(z^{1}, \ldots, z^{n}\right): K \rightarrow \mathbb{R}$ is called absolutely continuous function on $K$ if each of its coordinates functions $z^{i}, i=1, \ldots, n$, is absolutely continuous on $K$ in the sense of Definition 2.1.

The space of all absolutely continuous vector functions $z=\left(z^{1}, \ldots, z^{n}\right): K \rightarrow \mathbb{R}^{n}$ is denoted by $A C$. The norm in this space is defined by the formula

$$
\|z\|_{A C}=\left\|l^{1,2}\right\|_{L^{1}\left(K, \mathbb{R}^{n}\right)}+\left\|l^{1}\right\|_{L^{1}\left([0,1], \mathbb{R}^{n}\right)}+\left\|l^{2}\right\|_{L^{1}\left([0,1], \mathbb{R}^{n}\right)}+|c| .
$$

It is easy to see that the space $A C$ with this norm is a Banach space.

3. Families of equiabsolutely continuous functions of two variables; the AscoliArzela theorem. First, we recall some definitions.

A family $\left\{\varphi_{s}(\cdot), s \in S\right\}$ of functions defined on $[0,1](K)$ is called equibounded on $[0,1](K)$ if there exists some constant $R>0$ such that 


$$
\left|\varphi_{s}(t)\right| \leq R
$$

for all $t \in[0,1](t \in K)$ and $s \in S$.

A family $\left\{\varphi_{s}(\cdot), s \in S\right\}$ of absolutely continuous functions on $[0,1]$ is called equiabsolutely continuous on $[0,1]$ if, for any $\varepsilon>0$, there exists $\delta=\delta(\varepsilon)>0$ such that

$$
\sum_{i=1}^{N}\left|\varphi_{s}\left(\beta_{i}\right)-\varphi_{s}\left(\alpha_{i}\right)\right| \leq \varepsilon
$$

for all finite systems of nonoverlapping intervals $\left[\alpha_{i}, \beta_{i}\right], i=1, \ldots, N$, in $[0,1]$ with $\sum_{i=1}^{N}\left(\beta_{i}-\alpha_{i}\right)<\delta$ and for all $s \in S$.

A family $\left\{\varphi_{s}(\cdot), s \in S\right\}$ of integrable functions on $[0,1](K)$ is called equiabsolutely integrable on $[0,1](K)$ if, for any $\varepsilon>0$, there exists $\delta=\delta(\varepsilon)>0$ such that

$$
\int_{E}\left|\varphi_{s}\right| \leq \varepsilon
$$

for all measurable subsets $E$ of $[0,1](K)$ with $\mu_{1}(E) \leq \delta\left(\mu_{2}(E) \leq \delta\right)$ and for all $s \in S$, where $\mu_{1}$ denotes Lebesgue measure in $[0,1]$.

We have the following.

LEMMA 3.1. If $\left\{\varphi_{s}, s \in S\right\}$ is a family of absolutely continuous functions on [0,1], then this family is equiabsolutely continuous if and only if the family of derivatives $\left\{\varphi_{s}^{\prime}, s \in S\right\}$ is equiabsolutely integrable.

The above definitions and the proof of Lemma 3.1 can be found in [1, 10.2].

Now, let us introduce the notion of equiabsolute continuity of a family of absolutely continuous functions of an interval that are defined on the collection of all closed intervals contained in $K$.

So, a family $\left\{F_{S}: s \in S\right\}$ of functions of an interval, which are absolutely continuous on $K$, is called equiabsolutely continuous if, for any $\varepsilon>0$, there exists $\delta=\delta(\varepsilon)>0$ such that

$$
\sum_{i=1}^{N}\left|F_{S}\left(P_{i}\right)\right| \leq \varepsilon
$$

for all finite systems of nonoverlapping closed intervals $P_{i}, i=1, \ldots, N$, in $K$ with $\sum_{i=1}^{N} \mu_{2}\left(P_{i}\right) \leq \delta$ and for all $s \in S$.

Before we prove an analogue of Lemma 3.1 for functions of an interval, we recall (cf. [6]) that an absolutely continuous function $F$ on $K$ of an interval possesses a derivative $D F(x)$ for $x \in K$ a.e. This derivatives is integrable on $K$ and

$$
\int_{P} D F(x)=F(P)
$$

for any interval $P \subset K$.

LEMMA 3.2. If $\left\{F_{s}: s \in S\right\}$ is a family of functions of an interval, which are absolutely continuous on $K$, then this family is equiabsolutely continuous if and only if the family of derivatives $\left\{D F_{s}: s \in S\right\}$ is equiabsolutely integrable on $K$. 


\section{PROOF}

SuFfiCIENCY. Let us fix $\varepsilon>0$ and let $\delta>0$ be the number in the definition of equiabsolute integrability of the family of derivatives $\left\{D F_{s}, s \in S\right\}$. If $\left\{P_{i}, i=1, \ldots, N\right\}$ is a system of nonoverlapping closed intervals contained in $K$ with $\sum_{i=1}^{N} \mu_{2}\left(P_{i}\right) \leq \delta$, then

$$
\sum_{i=1}^{N}\left|F_{S}\left(P_{i}\right)\right|=\sum_{i=1}^{N}\left|\int_{P_{i}} D F_{S}\right| \leq \sum_{i=1}^{N} \int_{P_{i}}\left|D F_{S}\right|=\int_{\bigcup_{i=1}^{N} P_{i}}\left|D F_{S}\right| \leq \varepsilon
$$

for all $s \in S$ because $\mu_{2}\left(\bigcup_{i=1}^{N} P_{i}\right) \leq \delta$.

NeCESSITY. Let us fix $\varepsilon>0$ and let $\delta>0$ be the number in the definition of equiabsolute continuity on $K$ of the family $\left\{F_{s}, s \in S\right\}$ for $\varepsilon / 6$. Now, let us fix $s \in S$ and the set $E \subset K$ with $\mu_{2}(E) \leq \delta / 2$. Of course,

$$
\mu_{2}\left(E^{+}\right) \leq \frac{\delta}{2}, \quad \mu_{2}\left(E^{-}\right) \leq \frac{\delta}{2},
$$

where

$$
\begin{aligned}
& E^{+}=\left\{(x, y) \in E: D F_{S}(x, y) \geq 0\right\}, \\
& E^{-}=\left\{(x, y) \in E: D F_{S}(x, y) \leq 0\right\} .
\end{aligned}
$$

From the integrability of $D F_{s}$ it follows that there exists $\sigma>0$ (depending on $\varepsilon$ and $s$ ) such that

$$
\int_{F}\left|D F_{s}\right| \leq \frac{\varepsilon}{6}
$$

for any measurable set $F \subset K$ with $\mu(F) \leq \sigma$. Without loss of generality, we may assume that $\sigma \leq \delta / 2$.

Let $G$ be an open set such that

$$
E^{+} \subset G, \quad \mu_{2}(G) \leq \mu_{2}\left(E^{+}\right)+\sigma .
$$

From [6, Lemma V.4.1], it follows that there exists at most countable family $\left\{P_{i}, i=\right.$ $1,2, \ldots\}$ of disjoint right-hand open intervals $P_{i}=\left[x_{1}^{i}, x_{2}^{i}\left[\times\left[y_{1}^{i}, y_{2}^{i}[, i=1,2, \ldots\right.\right.\right.$, such that

$$
\bigcup_{i=1}^{\infty} P_{i}=G
$$

Consequently,

$$
\sum_{i=1}^{\infty} \mu_{2}\left(P_{i}\right)=\mu_{2}(G) \leq \mu_{2}\left(E^{+}\right)+\sigma \leq \frac{\delta}{2}+\frac{\delta}{2}=\delta
$$

If we denote

$$
G_{N}=\bigcup_{i=1}^{N} P_{i}
$$

for $N \in \mathbb{N}$, then we get

$$
\mu_{2}\left(G_{N}\right) \leq \delta
$$




$$
\begin{aligned}
\mu_{2}\left(G_{N} \backslash E^{+}\right) & \leq \mu_{2}\left(G \backslash E^{+}\right) \leq \mu_{2}(G)-\mu_{2}\left(G \cap E^{+}\right) \\
& \leq \mu_{2}\left(E^{+}\right)+\sigma-\mu_{2}\left(G \cap E^{+}\right) \\
& =\mu\left(E^{+}\right)+\sigma-\mu\left(E^{+}\right)=\sigma, \\
\mu_{2}\left(G \backslash G_{N}\right) & \leq \sigma,
\end{aligned}
$$

for sufficiently large $N$. Thus,

$$
\begin{aligned}
\int_{E^{+}}\left|D F_{s}\right| & =\int_{E^{+} \cap G} D F_{s}=\int_{E^{+} \cap G_{N}} D F_{s}+\int_{E^{+} \cap\left(G \backslash G_{N}\right)} D F_{s} \\
& =\left(\int_{E^{+} \cap G_{N}} D F_{s}+\int_{G_{N} \backslash E^{+}} D F_{S}\right)-\int_{G_{N} \backslash E^{+}} D F_{S}+\int_{E^{+} \cap\left(G \backslash G_{N}\right)} D F_{S} \\
& \leq \int_{G_{N}} D F_{S}+\int_{G_{N} \backslash E^{+}}\left|D F_{s}\right|+\int_{G \backslash G_{N}}\left|D F_{S}\right| \\
& \leq \sum_{i=1}^{N} F_{S}\left(\bar{P}_{i}\right)+\frac{\varepsilon}{6}+\frac{\varepsilon}{6} \leq 3 \cdot \frac{\varepsilon}{6}=\frac{\varepsilon}{2} .
\end{aligned}
$$

In an analogous way, we can show that

$$
\int_{E^{-}}\left|D F_{s}\right| \leq \frac{\varepsilon}{2} \text {. }
$$

So,

$$
\int_{E}\left|D F_{S}\right| \leq \varepsilon
$$

for any measurable set $E \subset K$ with $\mu_{2}(E) \leq \delta / 2$ and for any $s \in S$.

Now, let us introduce the notion of equiabsolute continuity of a family of absolutely continuous functions of two variables.

We say that a family $\left\{z_{s}, s \in S\right\}$ of functions of two variables, which are absolutely continuous on $K$, is equiabsolutely continuous if the families $\left\{F_{z_{s}}, s \in S\right\}$, $\left\{z_{s}(\cdot, 0), s \in S\right\}$, and $\left\{z_{s}(0, \cdot), s \in S\right\}$ are equiabsolutely continuous on $K,[0,1]$, and $[0,1]$, respectively.

Using equalities (2.1) and (2.2), we easily notice that, for an absolutely continuous function $z$,

$$
D F_{z}=\frac{\partial^{2} z}{\partial x \partial y}
$$

in $K$ a.e. From Lemmas 3.1 and 3.2, we immediately obtain

THEOREM 3.3. If $\left\{z_{s}, s \in S\right\}$ is a family of functions of two variables, which are absolutely continuous on $K$, then this family is equiabsolutely continuous if and only if the families $\left\{\partial^{2} z_{s} / \partial x \partial y, s \in S\right\},\left\{\partial z_{s} / \partial x(\cdot, 0), s \in S\right\}$, and $\left\{\partial z_{s} / \partial y(0, \cdot), s \in S\right\}$ are equiabsolutely integrable on $K,[0,1]$, and $[0,1]$, respectively.

We end the considerations of this section with Ascoli-Arzela theorem for absolutely continuous functions of two variables.

THEOREM 3.4. Let $\left(z_{n}\right)_{n \in \mathbb{N}}$ be a sequence of absolutely continuous functions on $K$. If it is equibounded and equiabsolutely continuous on $K$, then we can choose a 
subsequence $\left(z_{n_{k}}\right)_{k \in \mathbb{N}}$ that is uniformly convergent on $K$ to some function $z_{0}$, which is absolutely continuous on $K$.

Proof. It is easy to see that the equiabsolute continuity of the sequence $\left(z_{n}\right)_{n \in \mathbb{N}}$ carries its equicontinuity. Indeed, let $\varepsilon>0$ and $\delta=\min \left\{\delta_{1}, \delta_{2}, \delta_{3}\right\}$, where $\delta_{1}, \delta_{2}, \delta_{3}$ are the numbers in the definition of equiabsolute continuity of the sequences $\left(z_{n}(\cdot, 0)\right)_{n \in \mathbb{N}}$, $\left(z_{n}(0, \cdot)\right)_{n \in \mathbb{N}},\left(F_{z_{n}}\right)_{n \in \mathbb{N}}$, respectively, for $\varepsilon / 4$. Then, for any points $(\bar{x}, \bar{y}),(\overline{\bar{x}}, \overline{\bar{y}}) \in K$, with $|\bar{x}-\overline{\bar{x}}|+|\bar{y}-\overline{\bar{y}}|<\delta$, we have

$$
\begin{aligned}
\left|z_{n}(\bar{x}, \bar{y}),-z_{n}(\overline{\bar{x}}, \overline{\bar{y}})\right| \leq & \left|z_{n}(\bar{x}, \bar{y})-z_{n}(\bar{x}, \overline{\bar{y}})\right|+\left|z_{n}(\bar{x}, \overline{\bar{y}})-z_{n}(\overline{\bar{x}}, \overline{\bar{y}})\right| \\
\leq & \left|z_{n}(\bar{x}, \bar{y})-z_{n}(\bar{x}, \overline{\bar{y}})-z_{n}(0, \bar{y})+z_{n}(0, \overline{\bar{y}})\right| \\
& +\left|z_{n}(0, \bar{y})-z_{n}(0, \overline{\bar{y}})\right| \\
& +\left|z_{n}(\bar{x}, \overline{\bar{y}})-z_{n}(\overline{\bar{x}}, \overline{\bar{y}})-z_{n}(\bar{x}, 0)+z_{n}(\overline{\bar{x}}, 0)\right| \\
& +\left|z_{n}(\bar{x}, 0)-z_{n}(\overline{\bar{x}}, 0)\right| \\
\leq & 4 \cdot \frac{\varepsilon}{4}=\varepsilon
\end{aligned}
$$

for any $n \in \mathbb{N}$. Applying Ascoli-Arzela theorem for continuous functions (cf. [4, 1.5.4]), we assert that we can choose a subsequence $\left(z_{n_{k}}\right)_{k \in \mathbb{N}}$ that converges uniformly on $K$ to some function $z_{0}$ continuous on $K$.

Now, we show that the function $z_{0}$ is absolutely continuous on $K$. Indeed, from the equiabsolute continuity of the sequences $\left(F_{z_{n_{k}}}\right)_{k \in \mathbb{N}}$, we have for any $\varepsilon>0$, there exists $\delta>0$ such that

$$
\sum_{i=1}^{N}\left|F_{z_{n_{k}}}\left(P_{i}\right)\right| \leq \varepsilon
$$

for all finite systems of nonoverlapping closed intervals $P_{i} \subset K, i=1, \ldots, N$, with $\sum_{i=1}^{N} \mu_{2}\left(P_{i}\right)<\delta$ and for all $k \in \mathbb{N}$. If we denote $P_{i}=\left[x_{1}^{i}, x_{2}^{i}\right] \times\left[y_{1}^{i}, y_{2}^{i}\right], i=1, \ldots, N$, then inequality (3.21) can be written in the form

$$
\sum_{i=1}^{N}\left|z_{n_{k}}\left(x_{2}^{i}, y_{2}^{i}\right)-z_{n_{k}}\left(x_{1}^{i}, y_{2}^{i}\right)-z_{n_{k}}\left(x_{2}^{i}, y_{1}^{i}\right)+z_{n_{k}}\left(x_{1}^{i}, y_{1}^{i}\right)\right| \leq \varepsilon .
$$

Using the pointwise convergence of the sequence $\left(z_{n_{k}}\right)_{k \in \mathbb{N}}$ to $z_{0}$, we obtain from (3.22)

$$
\sum_{i=1}^{N}\left|z_{0}\left(x_{2}^{i}, y_{2}^{i}\right)-z_{0}\left(x_{1}^{i}, y_{2}^{i}\right)-z_{0}\left(x_{2}^{i}, y_{1}^{i}\right)+z_{0}\left(x_{1}^{i}, y_{1}^{i}\right)\right| \leq \varepsilon,
$$

i.e.,

$$
\sum_{i=1}^{N}\left|F_{z_{0}}\left(P_{i}\right)\right| \leq \varepsilon .
$$

This means that the function $F_{z_{0}}$ of an interval is absolutely continuous on $K$.

In an analogous way, we can show that the equiabsolute continuity of the sequence $\left(z_{n_{k}}(\cdot, 0)\right)_{k \in \mathbb{N}}$ implies the absolute continuity of the function $z_{0}(\cdot, 0)$, and the equiabsolute continuity of the sequence $\left(z_{n_{k}}(0, \cdot)\right)_{k \in \mathbb{N}}$ implies the absolute continuity of the function $z_{0}(0, \cdot)$.

So, the function $z_{0}$ is absolutely continuous on $K$ and the proof is completed. 
4. On the existence of an optimal solution. Let us consider system (1.3) and (1.4). In the sequel, we assume that the functions

$$
\begin{aligned}
& A_{0}: K \longrightarrow \mathbb{R}^{n, n}, \quad A_{1}: K \longrightarrow \mathbb{R}^{n, n}, \\
& A_{2}: K \longrightarrow \mathbb{R}^{n, n}, \quad B: K \longrightarrow \mathbb{R}^{n, r}
\end{aligned}
$$

are measurable and essentially bounded.

The class of admissible controls is defined as follows:

$u_{M}:=\left\{u: K \longrightarrow \mathbb{R}^{r} ; u\right.$ is measurable on $K$ and $u(x, y) \in M$ for $(x, y) \in K$ a.e. $\}$,

where $M \subset \mathbb{R}^{r}$ is a fixed compact and convex set.

In [11], the author proved the following.

THEOREM 4.1. For any control $u \in U_{M}$, there exists a unique solution $z \in A C$ of system (1.3) and (1.4) that satisfies (1.3) a.e. on $K$ and the boundary conditions (1.4) everywhere on $[0,1]$.

Since, in the sequel, we use some facts from the proof of Theorem 4.1, we reproduce the proof here.

Proof of Theorem 4.1. Let us define the following operator:

$$
\begin{aligned}
\mathscr{F} & : L^{1}\left(K, \mathbb{R}^{n}\right) \longrightarrow L^{1}\left(K, \mathbb{R}^{n}\right), \\
\mathscr{F}(l)(x, y)= & A_{0}(x, y) \int_{0}^{x} \int_{0}^{y} l(s, t) d t d s \\
& +A_{1}(x, y) \int_{0}^{y} l(x, t) d t+A_{2}(x, y) \int_{0}^{x} l(s, y) d s .
\end{aligned}
$$

It is easy to see that this operator is continuous. Consider a sequence $\left(l_{k}\right)_{k \in \mathbb{N}}$ defined by the recurrence relation

$$
l_{0}=0, \quad l_{k}=B u+\mathscr{F}\left(l_{k-1}\right), \quad k=1,2, \ldots .
$$

Of course, $l_{k}$ can be represented in the form

$$
l_{k}=\sum_{s=0}^{k-1} \mathscr{F}^{s}(B u),
$$

where

$$
\mathscr{F}^{0}(B u)=B u, \quad \mathscr{F}^{s}(B u)=\mathscr{F}\left(\mathscr{F}^{s-1}(B u)\right), \quad s=1,2, \ldots
$$

By definition,

$$
\mathscr{F}(B u)(x, y)=A_{0}(x, y) \int_{0}^{x} \int_{0}^{y} B u+A_{1}(x, y) \int_{0}^{y} B u+A_{2}(x, y) \int_{0}^{x} B u .
$$

So,

$$
|\mathscr{F}(B u)(x, y)| \leq 3 C N
$$


where

$$
\begin{aligned}
& C=\max _{i=0,1,2} \operatorname{ess} \sup _{(x, y) \in K}\left|A_{i}(x, y)\right|, \\
& N=\operatorname{ess}_{(x, y) \in K}|B(x, y)| \max _{u \in M}|u|
\end{aligned}
$$

and, consequently,

$$
\|\mathscr{F}(B u)\|_{L^{1}\left(K, \mathbb{R}^{n}\right)} \leq 3 C N .
$$

It can be easily noticed that $\mathscr{F}^{2}(\mathrm{Bu})$ is the sum of $3^{2}$ components, and that each component may be estimated by $C^{2} N$. Thus,

$$
\left|\mathscr{F}^{2}(B u)(x, y)\right| \leq(3 C)^{2} N \quad \text { for }(x, y) \in K \text { a.e. }
$$

and, consequently,

$$
\left\|\mathscr{F}^{2}(B u)\right\|_{L^{1}\left(K, \mathbb{R}^{n}\right)} \leq(3 C)^{2} N .
$$

On the basis of the induction principle, it can be shown that $\mathscr{F}^{s}(B u)$ is the sum of $3^{s}$ components. Each component of that sum is the product of $s$ coefficients $A_{i}, i=0,1,2$, and a $k$-fold, $k \geq s$, multiple integral. In this integral, there are at least $[(s+1) / 2]$ integrations with respect to $x$ or $y$. This implies that each component of the sum may be estimated by

$$
C^{s} N \frac{1}{[(s+1) / 2] !}
$$

Consequently,

$$
\left|\mathscr{F}^{s}(B u)(x, y)\right| \leq(3 C)^{s} N \frac{1}{[(s+1) / 2] !} \quad \text { for }(x, y) \in K \text { a.e. }
$$

So,

$$
\left\|\mathscr{F}^{s}(B u)\right\|_{L^{1}\left(K, \mathbb{R}^{n}\right)} \leq(3 C)^{s} N \frac{1}{[(s+1) / 2] !} .
$$

Since the series of numbers

$$
\sum_{s=0}^{\infty}(3 C)^{s} N \frac{1}{[(s+1) / 2] !}
$$

is convergent, there exists a limit (in $L^{1}\left(K, \mathbb{R}^{n}\right)$ )

$$
\lim _{k \rightarrow \infty} l_{k}=l_{u} \text {. }
$$

From the continuity of $\mathscr{F}$ and from (4.4), we obtain

$$
l_{u}=\mathscr{F}\left(l_{u}\right)+B u .
$$

Adopting

$$
z_{u}(x, y)=\int_{0}^{x} \int_{0}^{y} l_{u}(s, t) d s d t
$$

we obtain a solution of system (1.3) in the space $A C$, satisfying the boundary conditions (1.4). 
The fundamental role in this section is played by the following theorem.

THEOREM 4.2. Let $\left(u_{n}\right)_{n \in \mathbb{N}}$ be any sequence of elements of the set $u_{M}$ and $\left(z_{n}\right)_{n \in \mathbb{N}}-$ the sequence of the corresponding solutions of system (1.3) and (1.4) belonging to AC. There exist a control $u_{0} \in u_{M}$, a function $z_{0} \in A C$, and a subsequence $\left(n_{k}\right)_{k \in \mathbb{N}}$ of the sequence of positive integers, such that the pair $\left(z_{0}, u_{0}\right)$ satisfies system (1.3), (1.4), and

(i) $z_{n_{k}} \underset{k \rightarrow \infty}{\rightleftarrows} z_{0}$ uniformly on $K$;

(ii) $\partial^{2} z_{n_{k}} / \partial x \partial y \underset{k \rightarrow \infty}{\longrightarrow} \partial^{2} z_{0} / \partial x \partial y$ weakly in $L^{1}\left(K, \mathbb{R}^{n}\right)$;

(iii) $\partial z_{n_{k}} / \partial x \underset{k \rightarrow \infty}{\longrightarrow} \partial z_{0} / \partial x$ weakly in $L^{1}\left(K, \mathbb{R}^{n}\right)$;

(iv) $\partial z_{n_{k}} / \partial y \underset{k \rightarrow \infty}{\longrightarrow} \partial z_{0} / \partial y$ weakly in $L^{1}\left(K, \mathbb{R}^{n}\right)$;

(v) $u_{n_{k}} \underset{k \rightarrow \infty}{\longrightarrow} u_{0}$ weakly in $L^{1}\left(K, \mathbb{R}^{r}\right)$.

Before proving the above theorem, we give some lemmas. The first of them is a well-known result, so we give it without a proof.

LEMMA 4.3. From any sequence $\left(u_{n}\right)_{n \in \mathbb{N}}$ of elements of $u_{M}$, one can choose a subsequence $\left(u_{n_{k}}\right)_{k \in \mathbb{N}}$ such that $\left(u_{n_{k}}\right) \underset{k \rightarrow \infty}{\longrightarrow} u_{0}$ weakly in $L^{1}\left(K, \mathbb{R}^{r}\right)$, where $u_{0}$ is some element of $u_{M}$.

LEMMA 4.4. The family $\left\{\partial^{2} z_{u} / \partial x \partial y, u \in U_{M}\right\}$, where $z_{u}$ is the solution of the system (1.3) and (1.4) corresponding to a control $u \in u_{M}$, is equibounded on $K$.

Proof. Let us use the notation and some facts from the proof of Theorem 4.1. There, it was proved that, for any control $u \in U_{M}$,

$$
z_{u}(x, y)=\int_{0}^{x} \int_{0}^{y} l_{u}(s, t) d t d s
$$

Since, in view of (4.5) and (4.17),

$$
\frac{\partial^{2} z_{u}}{\partial x \partial y}(x, y)=l_{u}(x, y)=\sum_{s=0}^{\infty} \mathscr{F}^{s}(B u)(x, y),
$$

we have

$$
\left|\frac{\partial^{2} z_{u}}{\partial x \partial y}(x, y)\right| \leq \sum_{s=0}^{\infty}(3 C)^{s} N \frac{1}{[(s+1) / 2] !}<+\infty .
$$

The above sum does not depend on $u \in U_{M}$. Therefore, the proof is completed.

LEMMA 4.5. The family $\left\{z_{u}, u \in \mathcal{U}_{M}\right\}$ is equibounded on $K$.

Proof. The assertion follows directly from the equality

$$
z_{u}(x, y)=\int_{0}^{x} \int_{0}^{y} \frac{\partial^{2} z_{u}}{\partial x \partial y}(s, t) d t d s
$$

and Lemma 4.4.

From Lemma 4.4, we immediately get the following. 
LEMMA 4.6. The family $\left\{\partial^{2} z_{u} / \partial x \partial y, u \in U_{M}\right\}$ is equiabsolutely integrable on $K$.

Now, we give the proof of Theorem 4.2.

Proof. Let $\left(u_{n}\right)_{n \in \mathbb{N}}$ be a sequence of controls from $\varkappa_{M}$ and let us choose from it, on the basis of Lemma 4.3, a subsequence $\left(u_{n_{k}}\right)_{k \in \mathbb{N}}$ such that $u_{n_{k}} \underset{k \rightarrow \infty}{\longrightarrow} u_{0}$ weakly in $L^{1}\left(K, \mathbb{R}^{r}\right)$, where $u_{0}$ is some function belonging to $u_{M}$. From Lemmas 4.5 and 4.6, it follows that the sequence $\left(z_{n_{k}}\right)_{k \in \mathbb{N}}$ of the corresponding solutions of system (1.3) and (1.4) satisfies the assumptions of Theorem 3.4. So, we may choose a subsequence, say still $\left(n_{k}\right)$, such that $z_{n_{k}} \underset{k \rightarrow \infty}{\rightrightarrows} z_{0}$ uniformly on $K$, where $z_{0}$ is some function from $A C$. From Lemma 4.6, it follows that the sequence $\left(\partial^{2} z_{n_{k}} / \partial x \partial y_{k \in \mathbb{N}}\right)$ is equiabsolutely integrable on $K$. Thus, making use of Dunford-Pettis theorem (cf. [1, 10.3(i)]), we may choose a subsequence, say still $\left(n_{k}\right)_{k \in \mathbb{N}}$, such that $\partial^{2} z_{n_{k}} / \partial x \partial y \underset{k \rightarrow \infty}{\longrightarrow} \sigma$ weakly in $L^{1}\left(K, \mathbb{R}^{n}\right)$, where $\sigma$ is some function from $L^{1}\left(K, \mathbb{R}^{n}\right)$. In view of the above, let us observe that, for any $(x, y) \in K$,

$$
\begin{aligned}
z_{n_{k}}(x, y) & =\int_{0}^{x} \int_{0}^{y} \frac{\partial^{2} z_{n_{k}}}{\partial x \partial y} \\
& =\int_{0}^{1} \int_{0}^{1} x_{[0, x] \times[0, y]} \frac{\partial^{2} z_{n_{k}}}{\partial x \partial y} \underset{k \rightarrow \infty}{\longrightarrow} \int_{0}^{1} \int_{0}^{1} x_{[0, x] \times[0, y]} \sigma=\int_{0}^{x} \int_{0}^{y} \sigma .
\end{aligned}
$$

$\chi_{A}$ denotes the characteristic function of the set $A$.

On the other hand, since the sequence $\left(z_{n_{k}}\right)_{k \in \mathbb{N}}$ converges uniformly on $K$ to $z_{0}$, we have

$$
z_{n_{k}}(x, y) \underset{k \rightarrow \infty}{\longrightarrow} z_{0}(x, y)
$$

for any $(x, y) \in K$. Consequently,

$$
z_{0}(x, y)=\int_{0}^{x} \int_{0}^{y} \sigma
$$

for any $(x, y) \in K$, and

$$
\frac{\partial^{2} z_{0}}{\partial x \partial y}(x, y)=\sigma(x, y)
$$

for $(x, y) \in K$ a.e. Thus,

$$
\frac{\partial^{2} z_{n_{k}}}{\partial x \partial y} \underset{k \rightarrow \infty}{\longrightarrow} \frac{\partial^{2} z_{0}}{\partial x \partial y}
$$

weakly in $L^{1}\left(K, \mathbb{R}^{n}\right)$.

Now, let us observe that

$$
\frac{\partial z_{n_{k}}}{\partial x}(x, y)=\int_{0}^{y} \frac{\partial^{2} z_{n_{k}}}{\partial x \partial y}(x, t) d t
$$

for $(x, y) \in K$ a.e., and that

$$
\frac{\partial z_{n_{k}}}{\partial y}(x, y)=\int_{0}^{x} \frac{\partial^{2} z_{n_{k}}}{\partial x \partial y}(s, y) d s
$$


for $(x, y) \in K$ a.e. So, from the linearity and the continuity of the operator

$$
\begin{aligned}
\mathscr{L}: L^{1}\left(K, \mathbb{R}^{n}\right) & \longrightarrow L^{1}\left(K, \mathbb{R}^{n}\right), \\
\mathscr{L}(g)(x, y) & =\int_{0}^{y} g(x, t) d t,
\end{aligned}
$$

and from the fact that the sequence $\left(\partial^{2} z_{n_{k}} / \partial x \partial y\right)_{k \in \mathbb{N}}$ converges weakly in $L^{1}\left(K, \mathbb{R}^{n}\right)$ to $\partial^{2} z_{0} / \partial x \partial y$, we obtain (cf. [7, III.24.3])

$$
\frac{\partial z_{n_{k}}}{\partial x} \underset{k \rightarrow \infty}{\longrightarrow} \frac{\partial z_{0}}{\partial x}
$$

weakly in $L^{1}\left(K, \mathbb{R}^{n}\right)$. In an analogous way, we assert that

$$
\frac{\partial z_{n_{k}}}{\partial y} \underset{k \rightarrow \infty}{\rightarrow} \frac{\partial z_{0}}{\partial y}
$$

weakly in $L^{1}\left(K, \mathbb{R}^{n}\right)$.

To complete the proof, it is sufficient to show that the pair $\left(z_{0}, u_{0}\right)$ satisfies system (1.3) and (1.4).

Indeed, the fact that $z_{0}$ satisfies the boundary conditions (1.4) follows immediately from the uniform convergence of the sequence $\left(z_{n_{k}}\right)_{k \in \mathbb{N}}$ to $z_{0}$.

The fact that $\left(z_{0}, u_{0}\right)$ satisfies (1.3) follows from the convergences

$$
\frac{\partial^{2} z_{n_{k}}}{\partial x \partial y} \underset{k \rightarrow \infty}{\longrightarrow} \frac{\partial^{2} z_{0}}{\partial x \partial y}
$$

weakly in $L^{1}\left(K, \mathbb{R}^{n}\right)$,

$$
A_{0}(\cdot, \cdot) z_{n_{k}} \underset{k \rightarrow \infty}{\longrightarrow} A_{0}(\cdot, \cdot) z_{0}
$$

weakly in $L^{1}\left(K, \mathbb{R}^{n}\right)$,

$$
A_{1}(\cdot, \cdot) \frac{\partial z_{n_{k}}}{\partial x} \underset{k \rightarrow \infty}{\longrightarrow} A_{1}(\cdot, \cdot) \frac{\partial z_{0}}{\partial x}
$$

weakly in $L^{1}\left(K, \mathbb{R}^{n}\right)$,

$$
A_{2}(\cdot, \cdot) \frac{\partial z_{n_{k}}}{\partial y} \underset{k \rightarrow \infty}{\longrightarrow} A_{2}(\cdot, \cdot) \frac{\partial z_{0}}{\partial y}
$$

weakly in $L^{1}\left(K, \mathbb{R}^{n}\right)$,

$$
B(\cdot, \cdot) u_{n_{k}} \underset{k \rightarrow \infty}{\longrightarrow} B(\cdot, \cdot) u_{0}
$$

weakly in $L^{1}\left(K, \mathbb{R}^{n}\right)$ and from the fact that each pair $\left(z_{n_{k}}, u_{n_{k}}\right), k \in \mathbb{N}$, satisfies (1.3).

Now, let us consider Bolza problem (1.3), (1.4), and (1.5) in the spaces $A C$ of trajectories and $U_{M}$ of controls. Consider the function

$$
f^{0}: K \times \mathbb{R}^{n} \times \mathbb{R}^{r} \longrightarrow \mathbb{R}
$$

We assume that 
(1) for any $z \in \mathbb{R}^{n}, u \in \mathbb{R}^{r}$, the function $f^{0}(\cdot, \cdot, z, u)$ is measurable on $K$,

(2) for any $(x, y) \in K$, the function $f^{0}(x, y, \cdot, \cdot)$ is continuous on $\mathbb{R}^{n} \times \mathbb{R}^{r}$,

(3) for any $(x, y) \in K, z \in \mathbb{R}^{n}$, the function $f^{0}(x, y, z, \cdot)$ is convex on $\mathbb{R}^{r}$,

(4) there exist a function $\psi: K \rightarrow \mathbb{R}_{0}^{+}$belonging to $L^{1}(K, \mathbb{R})$ and a constant $c \geq 0$ such that

$$
f^{0}(x, y, z, u) \geq-\psi(x, y)-c|u|
$$

for $(x, y) \in K$ a.e. and $z \in \mathbb{R}^{n}, u \in \mathbb{R}^{r}$.

We denote

$$
m=\inf \left\{I\left(z_{u}, u\right), u \in \cup_{M}\right\} .
$$

From (4), it follows that $-\infty<m \leq+\infty$.

When $m=+\infty$, the existence of an optimal solution is obvious.

So, let us assume that $-\infty<m<+\infty$. Let $\left(z_{n}, u_{n}\right)_{n \in \mathbb{N}}$ be a minimizing sequence for the functional $I$, i.e.,

$$
m=\lim _{n \rightarrow \infty} I\left(z_{n}, u_{n}\right)
$$

where $z_{n}=z_{u_{n}}$.

Making use of Theorem 4.2, we assert that there exist a pair $\left(z_{0}, u_{0}\right) \in A C \times{u_{M}}_{M}$ and a subsequence $\left(n_{k}\right)_{k \in \mathbb{N}}$ of the sequence of positive integers, such that the pair $\left(z_{0}, u_{0}\right)$ satisfies system (1.3), (1.4), and

$$
z_{n_{k}} \underset{k \rightarrow \infty}{\longrightarrow} z_{0}
$$

uniformly on $K$,

$$
u_{n_{k}} \underset{k \rightarrow \infty}{\longrightarrow} u_{0}
$$

weakly in $L^{1}\left(K, \mathbb{R}^{r}\right)$. Thus, from $[1,10.8(i)]$, we obtain

$$
m \leq I\left(z_{0}, u_{0}\right) \leq \liminf _{k \rightarrow \infty} I\left(z_{n_{k}}, u_{n_{k}}\right)=\lim _{k \rightarrow \infty} I\left(z_{n_{k}}, u_{n_{k}}\right)=m .
$$

Hence,

$$
I\left(z_{0}, u_{0}\right)=\inf \left\{I\left(z_{u}, u\right), u \in U_{M}\right\} .
$$

So, we have proved the following theorem.

THEOREM 4.7. If conditions (4.1), (1), (2), (3), and (4) are satisfied, and the set $M \subset \mathbb{R}^{r}$ is compact and convex, then there exists an optimal solution $\left(z_{0}, u_{0}\right)$ of Bolza problem (1.3), (1.4), and (1.5) in the spaces AC of trajectories and $u_{M}$ of controls.

5. On some physical interpretation. Let us consider a gas filter made in the form of a pipe filled up with a substance $S$ which absorbs a poison gas. Through the filter, a mixture of air and gas is pressed at a speed $v=v(x, t)>a>0$ with the aid of an aggregation $A$. We denote by $y=y(x, t)$ the quantity of the poison gas being present in the capacity unit of the substance $S$ at a distance $x$ from the inlet of the filter and at a moment $t$. Assume that the speed $v=v(x, t)$ is so great that the diffusion 
process plays no essential role in the motion of the gas. In this case, the process of the absorption of the poison gas by the filter, filled up with the substance $S$, is described by a differential equation of the form

$$
\frac{\partial^{2} y}{\partial x \partial t}(x, t)+\frac{\beta}{v(x, t)} \frac{\partial y}{\partial t}(x, t)+\beta \gamma \frac{\partial y}{\partial x}(x, t)=0
$$

under the boundary conditions

$$
y(x, 0)=y_{0} \exp \left(-\frac{\beta}{v_{0}} x\right), \quad y(0, t)=y_{0},
$$

where $y_{0}$ is the gas concentration at the inlet to the filter ( $y_{0}$-const.), $v(x, t)$ denotes the speed of the flow of the mixture of air and gas through the filter at the moment $t$ and the distance $x$ from the inlet of the filter, $v_{0}=v(0,0), \beta$ and $\gamma$ are physical quantities characterizing the given gas (for details, see [10, Chapter II]).

Without loss of generality, we may assume that $x \in[0,1]$ and $t \in[0,1]$. Put

$$
y(x, t)=z(x, t)+y_{0} \exp \left(-\frac{\beta}{v_{0}} x\right)
$$

It is easy to demonstrate that the system (5.1) and (5.2) is equivalent to a system of the form

$$
\begin{gathered}
\frac{\partial^{2} z}{\partial x \partial t}(x, t)+\beta \gamma \frac{\partial z}{\partial x}(x, t)+\frac{\beta}{v(x, t)} \frac{\partial z}{\partial t}(x, t)-\frac{\gamma \beta^{2} y_{0}}{v_{0}} \exp \left(-\frac{\beta}{v_{0}} x\right)=0, \\
z(x, 0)=0, \quad z(0, t)=0 .
\end{gathered}
$$

Let us suppose that we have some influence on the process of the filtering of the gas, and that our control has a linear character. In this situation, we can assume that the system describing this process is of the form

$$
\begin{gathered}
\frac{\partial^{2} z}{\partial x \partial t}(x, t)+\beta \gamma \frac{\partial z}{\partial x}(x, t)+\frac{\beta}{v(x, t)} \frac{\partial z}{\partial t}(x, t)=\frac{\gamma \beta^{2} y_{0}}{v_{0}} \exp \left(-\frac{\beta}{v_{0}} x\right) u(x, t), \\
z(x, 0)=0, \quad z(0, t)=0 .
\end{gathered}
$$

The function $u: K \rightarrow[c, d]$, where $-\infty<c<d<\infty$ are fixed numbers, is treated as a control. Suppose that the cost functional has the form

$$
I(z, u)=\int_{0}^{1} \int_{0}^{1} f^{0}(x, t, z(x, t), u(x, t)) d x d t .
$$

Assume that $f^{0}$ satisfies conditions (1), (2), (3), and (4). By Theorem 4.7, control system (5.5), (5.6), and (5.7) possesses an optimal process $\left(z_{0}, u_{0}\right)$ in the space of absolutely continuous trajectories $z \in A C$ and in the set of admissible controls $u \in L^{\infty}([0,1],[c, d])$.

ACKNOWLEDGEMENT. Supported by Grant 211029101 of the State Committee for Scientific Research. 


\section{REFERENCES}

[1] L. Cesari, Optimization-Theory and Applications, Springer-Verlag, New York, 1983. MR 85c:49001. Zbl 506.49001.

[2] Z. Denkowski and A. Pelczar, On the existence and uniqueness of solutions of some partial differential functional equations, Ann. Polon. Math. 35 (1977/78), no. 3, 261-304. MR 58\#12030. Zbl 378.35002.

[3] A. I. Egorov, Necessary optimal conditions for systems with distributed parameters, Mat. Sb. (N.S.) 69 (111) (1966), no. 3, 371-421 (Russian). MR 33\#2443. Zbl 163.11305.

[4] L. V. Kantorovich and G. P. Akilov, Functional Analysis, "Nauka”, Moscow, 1984 (Russian). MR 86m:46001. Zbl 555.46001.

[5] E. B. Lee and L. Markus, Foundations of Optimal Control Theory, John Wiley \& Sons Inc., New York, 1967. MR 36\#3596. Zbl 159.13201.

[6] S. Łojasiewicz, An Introduction to the Theory of Real Functions, John Wiley \& Sons Ltd., Chichester, 1988. MR 89e:26001. Zbl 653.26001.

[7] L. A. Lusternik and W. I. Sobolev, Elements of Functional Analysis, Hindustan Publishing Corp., Delhi, 1974. MR 50\#2852. Zbl 293.46001.

[8] L. I. Rozonoèr, L. S. Pontryagin maximum principle in the theory of optimum systems. I, II, III, Automat. Remote Control 20 (1959), no. 10, 1288-1302, 1405-1421, 15171532. MR 22\#11193. Zbl 124.05801.

[9] M. B. Suryanarayana, On multidimensional integral equations of Volterra type, Pacific J. Math. 41 (1972), no. 3, 809-828. MR 48\#2693. Zbl 241.45005.

[10] A. N. Tijonov and A. A. Samarsky, Ecuaciones de la física matemática, "Mir", Moscow, 1983. MR 85c:35001.

[11] S. Walczak, Absolutely continuous functions of several variables and their application to differential equations, Bull. Polish Acad. Sci. Math. 35 (1987), no. 11-12, 733-744. MR 90d:26026. Zbl 691.35029.

[12] - On the Differentaiablity of Absolutely Continuous Functions of Several Variables, ibid. 36 (1988), no. 9-10, 513-520. Zbl 762.26010.

[13]___ Optimality conditions for a Bolza problem governed by a hyperbolic system of Darboux-Goursat type, Ann. Polon. Math. 53 (1991), no. 1, 7-14. MR 92c:49015. Zbl 742.49018 .

IdCZAK AND WALCZAK: InSTITUTE OF MATHEMATICS, ŁóDŹ UNIVERSiTy, Ul. STEFANA BANACHA 22, 90-238 ŁóDź, POLAND 


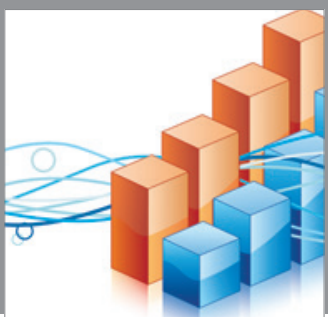

Advances in

Operations Research

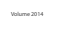

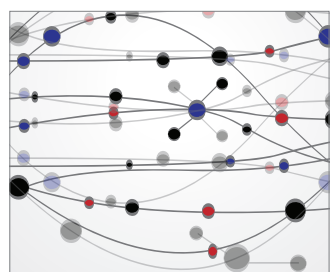

\section{The Scientific} World Journal
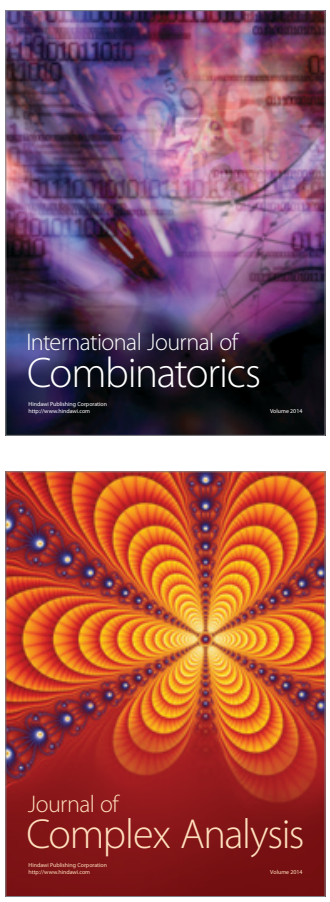

International Journal of

Mathematics and

Mathematical

Sciences
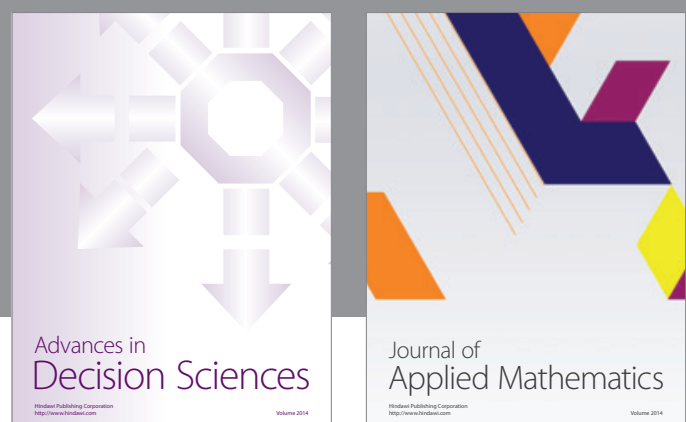

Journal of

Applied Mathematics
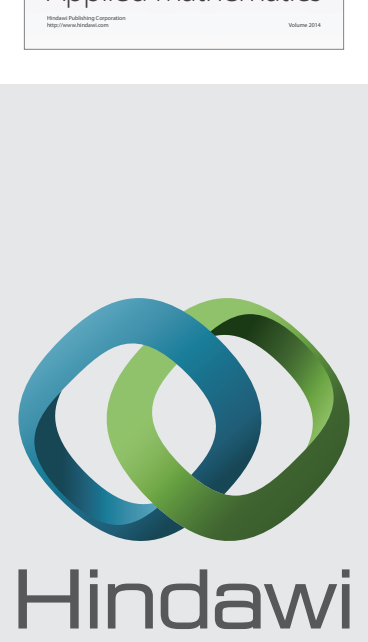

Submit your manuscripts at http://www.hindawi.com
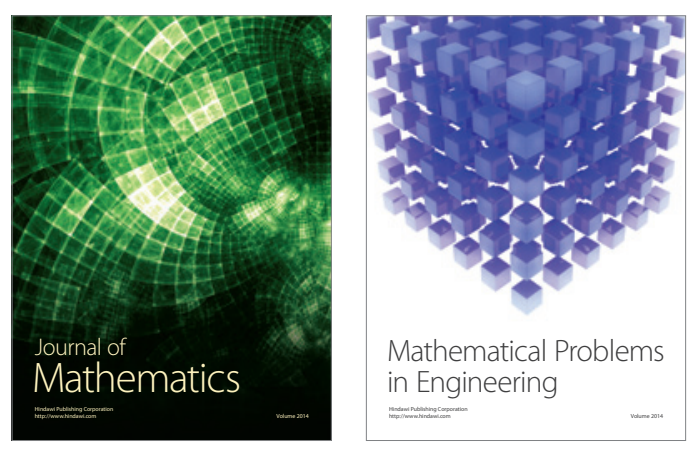

Mathematical Problems in Engineering
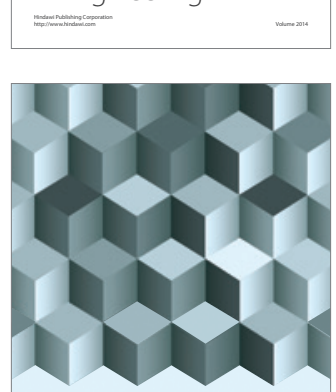

Journal of

Function Spaces
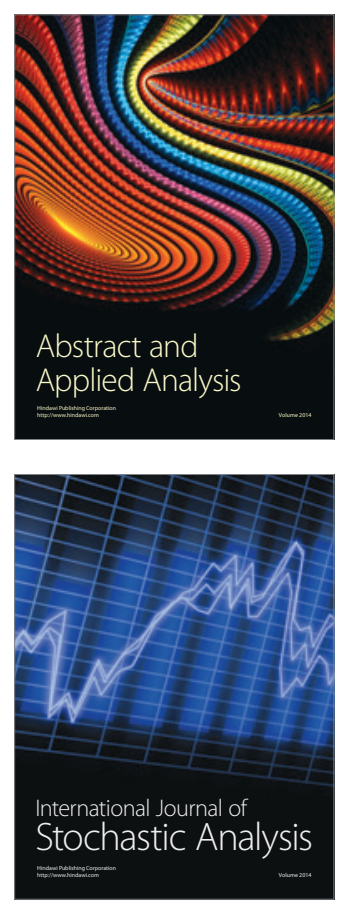

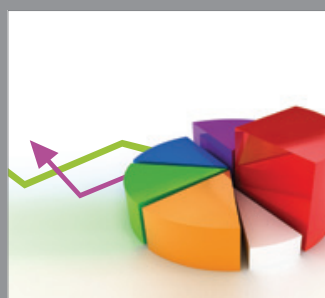

ournal of

Probability and Statistics

Promensencen
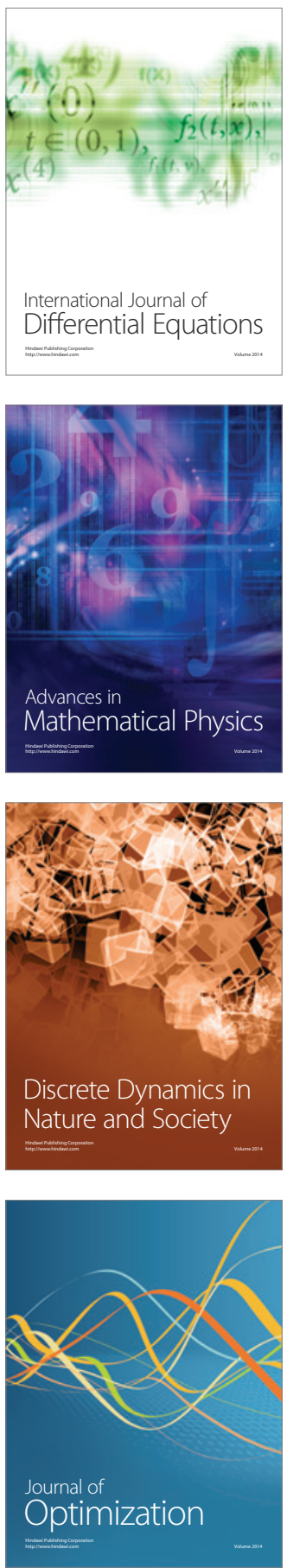